\title{
Primary synovial sarcoma of the kidney: a case report of complete pathological response at a Lebanese tertiary care center
}

\author{
Alissar El Chediak', Deborah Mukherji ${ }^{1}$, Sally Temraz ${ }^{1}$, Samer Nassif ${ }^{2}$, Sara Sinno², Rami Mahfouz ${ }^{2}$ \\ and Ali Shamseddine ${ }^{1 *}$ (D)
}

\begin{abstract}
Background: Primary synovial sarcoma of the kidney is a rare type of soft tissue sarcoma. Its presenting features can resemble those of other renal tumors; rendering its early diagnosis, a dilemma. Several cases of renal synovial sarcoma have been reported in the literature with varying treatment options and outcomes. This article describes a rare case of primary renal synovial sarcoma and reviews all cases in the literature.

Case presentation: A 26-year-old male presented with flank pain and hematuria. Initially diagnosed with Wilm's tumor, revision of pathology and histology, along with the immunohistochemical profile, confirmed, nevertheless, the diagnosis of primary monophasic synovial sarcoma of the kidney with the SYT-SSX2 fusion transcript. Follow-up, post nephrectomy, revealed recurrence within the lungs and at the surgical bed. Surgical resection followed by adjuvant chemotherapy regimen constituting of Doxorubicin and Ifosfamide, achieved complete pathological response.

Conclusion: In this case report, we emphasize the need for accurate diagnosis and prompt treatment. We propose multimodality treatment approach including surgery along with anthracycline-based chemotherapy to induce complete remission.
\end{abstract}

Keywords: Synovial sarcoma, SYT-SSX, Doxorubicin, Ifosfamide, Pathological response, Survival

\section{Background}

Soft tissue sarcoma (STS) is a rare malignant tumor of mesenchymal origin having an incidence of $2-3$ cases per 100,000 , thus contributing to less than $1 \%$ of all adult malignancies [1, 2]. Synovial sarcoma (SS), or sarcoma of tissues adjacent to joints, is a rare type of STS, and represent 5 to10\% of all STSs [1]. SS is commonly found in the proximal limb of young adults and has a male predominance [3]. Other unusual sites of occurrence include the head and neck, heart, lungs, and kidneys [4]. Very few reports have tackled this tumor due to its rarity and difficulty to distinguish from other renal pathologies. The first case of primary SS of the kidney has been reported by Faria et al. in 1999 [5]. We present a case of primary synovial sarcoma of the kidney, initially thought to be a

\footnotetext{
* Correspondence: as04@aub.edu.lb

${ }^{1}$ Department of Internal Medicine, Division of Hematology/Oncology,

American University of Beirut - Medical Center, P.o.Box: 11-0236, Riad El Solh,

Beirut 110 72020, Lebanon

Full list of author information is available at the end of the article
}

Wilm's tumor, along with patient follow-up, showing complete pathological response to treatment, followed by a literature review of this disease entity.

\section{Case presentation}

A 26-year-old male experienced recurrent flank pain and gross hematuria over several months duration. Kidney ultrasound showed a lower pole mass concerning for renal cell carcinoma. After confirmation of a right kidney tumor, measuring $6 \mathrm{~cm}$, by an enhanced CT scan, he underwent right radical nephrectomy with para-caval lymph node dissection, at another institute, with pathology there, read initially as adult type Wilm's tumor. After referral to our institute for rereading of the pathological slides, the morphological and immunostaining profiles were analyzed, and results came out to be consistent with synovial sarcoma of the right kidney. The tumor was monophasic and showed a cellular spindle cell proliferation with a prominent perivascular growth 
pattern and partial necrosis. It was positive for vimentin, BCL-2, CD56, MCK (partial), and negative for CD10, 31, 34, 99, 117, CK7, Desmin, SMA, MyoD1, EMA, WT-1, S100, RCC, PAX8, GATA-3, and Synaptophysin (Fig. 1).

Molecular studies on the paraffin-embedded blocks were performed to test for the $t(X ; 18)$ SYT/SSX fusion transcript, using RT-PCR, at the University of Michigan Health System. RT-PCR amplification was performed using fluorescent dye-labeled primers, specific for the SYT-SS18 and SYT-SSX genes. The PCR products were then detected and sized by capillary electrophoresis to identify the presence of chimeric transcripts. A concurrent internal control was run to ensure the integrity of the mRNA. FISH analysis was also performed using a break-apart style probe. The results were unfortunately negative due to the low quality samples.

According to these findings, a diagnosis of primary monophasic SS of the kidney was made. It was elected for serial follow up and no adjuvant treatment, thereafter. Six months later, a follow up CT scan detected a 1. $5 \mathrm{cmx} 1.7 \mathrm{~cm}$ left lower lobe lung nodule suggestive of metastasis. Consequently, he underwent a smooth left lower lobe wedge resection. Fusion gene product analysis on the resected lung tissue, via FISH, revealed SYTSSX 2 gene rearrangement confirming the SS diagnosis. Three months afterwards, CT scan of the chest, abdomen, and pelvis revealed another disease recurrence in the nephrectomy surgical bed, with tumor invasion of the inferior vena cava and the presence of conglomerate suspicious aorto-iliac lymph nodes. A multidisciplinary team approach decided to start the patient on Doxorubicin $50 \mathrm{~g} / \mathrm{m}^{2}$ and Ifosfamide $5 \mathrm{~g} / \mathrm{m}^{2}$ chemotherapeutic regimen. Following the third cycle, CT scan and MRI showed a 30 to $50 \%$ interval decrease in size of tumor masses in the right nephrectomy bed and adjacent retroperitoneum, IVC tumor, and distal aortocaval lymph nodes, indicating partial treatment response. The patient were received a total of 5 cycles, with no adjunct side effects.

A follow-up MRI, several months later, showed continued decrease in the size of 3 masses at the previous surgical site, IVC tumor invasion, and aortocaval lymph nodes, indicating continued response to treatment. One of the small masses in the nephrectomy bed almost completely resolved, on imaging, with no new progression. It was then decided to have the patient undergo surgical resection of the residual masses at the previous surgical bed with removal of the aorto-caval lymph nodes, thrombectomy with vena caval repair. All surgical margins were negative. Final pathology came out to be necrosis, with no viable tumor identified. Thus, a complete pathological response was achieved using the Adriamycin/Ifosfamide regimen, a year after the initial nephrectomy. A sample of the kidney lysate was again tested for the $(\mathrm{X} ; 18)$ SYT/SSX fusion transcript via RTPCR and FISH, and results were negative, suggestive of complete treatment response.

\section{Discussion}

Synovial sarcoma is a mesenchymal spindle cell tumor which displays variable epithelial differentiation and has a specific chromosomal translocation $t(X ; 18)(p 11 ; q 11)$, which results from the fusion of the SYT gene on chromosome 18 to exon 5 of either SSX1 or SSX2 genes

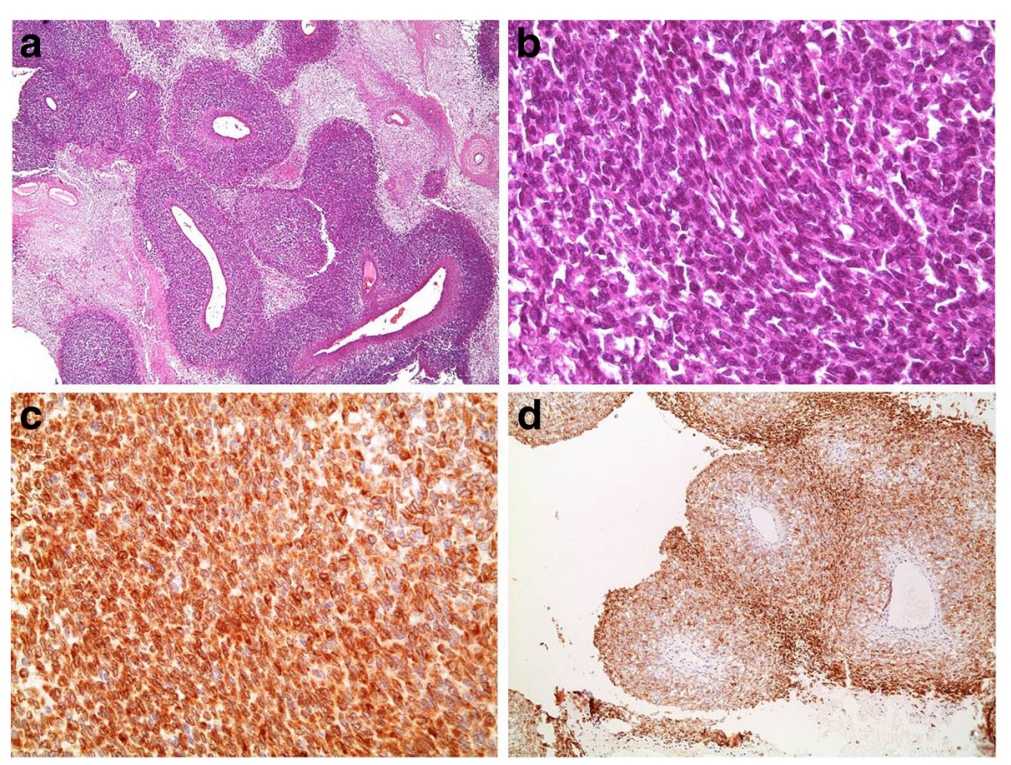

Fig. 1 Partially necrotic, densely cellular proliferation with a prominent perivascular growth pattern (a, H\&E stain, 40X). Tumor cells are essentially spindle in appearance (b, H\&E stain, 400x), and express vimentin (not shown), focal keratin (not shown), BCL-2 (c, 400x), and CD56 (d, 100X) 
on chromosome X [6]. It was recently reported that the SSX4 gene is also involved in such a translocation $[6,7]$. Nonetheless, SS of the kidney can be first misdiagnosed as a renal cell carcinoma due to similar clinical presentation [3]. The identification of the monophasic type of renal SS is also controversial as it has similar microscopic features to other spindle cell tumors, such as fibrosarcoma, leiomyosarcoma, malignant peripheral nerve sheath tumors, adult Wilm's tumor, spindle cell carcinoma and spindle cell melanoma [3]. While monophasic SS of the kidney is made up exclusively of monomorphic spindle cells, the biphasic type is a mixture of both spindle-shaped cells and epithelial cells. SS of the kidney is a rare disease such as 64 cases have been reported since the Faria et al. cases up to 2012 [6]. We conducted a literature review using Embase and PubMed databases and included all cases (even those published in languages other than English) till the year 2016. This yielded a total of 114 cases (Table 1) constituting the largest series of renal SS cases to be reported. Noteworthy, our case is the first to be reported from the Middle East.

The median age of patients with renal SS was 40.5 (15-78) years, which is half the median age for diagnosis of renal cell carcinoma [7]. The female to male ratio was 1:1. Regarding the predominant presentation symptoms, data was only available for 82 cases. The most frequently reported symptom on presentation was isolated flank/ lumbar pain which was found in 20 patients (24.4\%).This is in concordance with a review conducted on older data where this same predominant symptom occurred in 55 . $5 \%$ of cases [8]. Hematuria was present in 37 patients (44\%) upon presentation. Kohle et al. reported similar data, albeit smaller sample size, where $98 \%$ of their patients were symptomatic at the time of presentation, with $67 \%$ having pain and 38\% having hematuria [6]. These figures are in concordance with our analysis of the world literature.

According to our analysis, the leading fusion variant was the SYT-SSX 2, detected in $42(36.8 \%)$ patients, as opposed to the SYT-SSX 1 variant, detected in $23(20.2 \%)$ patients, in total (Table 1).

Data on metastasis and disease recurrence was available for 70 and 57 patients, respectively. 19 (27.1\%) patients had metastasis, whereas $14(24.6 \%)$ patients had tumor recurrence on follow-up. These numbers are similar to what was published in previous series [9].

A 30 to $50 \%$ of the patients who underwent surgical resection of their primary tumor were reported to witness metastasis to the lungs or liver [10]. Our patient is of no exception. In terms of median survival for patients with localized disease, size represents an important prognostic factor [10]. A retrospective analysis was done on 135 consecutive patients with extremity and truncal variant of synovial sarcomas, seen at three institutions in
Boston, between years 1961 and 1996. Patients with localized synovial sarcomas, less than $5 \mathrm{~cm}$ in longest diameter, had a survival at 10 years equivalent to $88 \%$, compared with a 10-year survival of 38 and $8 \%$ for 5 to $10 \mathrm{~cm}$ and greater than $10 \mathrm{~cm}$ sarcomas, respectively [10]. Similar results were reported by Singer et al. on 48 consecutive patients with extremity and truncal synovial sarcomas, seen between years 1966 and 1994 [11].

SS of the kidneys presents a diagnostic dilemma, as it resembles renal cell carcinoma amongst other tumors. Diagnosis can be based on morphological (spindle cell) and immunological tumor profile. It can also be established by genetic analysis via FISH and RT-PCR, demonstrating the SYT18-SSX gene translocation. Demonstrating which translocation, the tumor possesses, has been a delicate matter. For instance, a SS case that was previously shown to be negative for SYT/SSX1 and SYT/SSX2 gene expression by conventional RT-PCR, was instead found to be SYT/SSX4 positive, as the sole fusion transcript expressed in this tumor sample, when the RT-PCR was redesigned [6]. This might have explained our negative results, when trying to identify the translocations, since our RT-PCR was only designed to detect SYT/SSX1 and SYT/SSX2 fusion gene variants.

Moreover, the value of TLE1 antibody in the diagnosis of SS has also been examined. TLE1 was found to be an excellent discriminator of SS from other sarcomas, in a study by Terry et al. [12]. They reported that TLE1 monoclonal and polyclonal antibodies gave intense and/ or diffuse nuclear staining in 91 out of 94 molecularly confirmed synovial sarcoma patients. In contrast, TLE1 staining has been detected much less frequently and at lower levels, if et al.l, in 40 other mesenchymal tumors; thereby making this a robust immunohistochemical marker for SS. Jagdis et al. also supported this view as their findings confirmed that TLE1 was more sensitive and specific for synovial sarcoma, than any other currently available immunohistochemical kits [13]. Kosemehmetoglu et al. confirmed the sensitivity, but not the specificity, of TLE1 antibodies in diagnosing synovial sarcomas [14].

The prognostic implication of SYT-SSX fusion type in synovial sarcomas is still under debate. The SYT-SSX fusion type and the presence of metastasis, at diagnosis, were both proven to be important prognostic indicators [15]. Kawai et al. analyzed SYT-SSX fusion transcripts in 45 synovial sarcomas by reverse-transcriptase polymerase chain reaction, and compared the results with relevant clinical and pathological data [16]. SYT- SSX2 fusion type carried a significant positive prognosis for overall survival [16]. This was thought to be due to an association with a lower prevalence of metastatic disease at diagnosis, in patients having this rearrangement [16]. In another study by Ladanyi et al., the SYT-SSX2 fusion 
Table 1 List of 114 cases of renal SS published in the literature

\begin{tabular}{|c|c|c|c|c|c|c|c|}
\hline $\begin{array}{l}\text { Case } \\
\text { Report/ } \\
\text { Series }\end{array}$ & $\begin{array}{l}\text { Author/Year } \\
\text { of publication }\end{array}$ & $\begin{array}{l}\text { No of } \\
\text { cases }\end{array}$ & $\begin{array}{l}\text { Age(Y)/Gender } \\
(\mathrm{M} / \mathrm{F})\end{array}$ & $\begin{array}{l}\text { Presenting } \\
\text { symptoms }\end{array}$ & $\begin{array}{l}\text { Fusion gene } \\
\text { Variant }\end{array}$ & Treatment & Outcome \\
\hline 1 & $\begin{array}{l}\text { Argani P et al.; } \\
2000 \text { [5] }\end{array}$ & 17 & $\begin{array}{l}10 \mathrm{M} \\
7 \mathrm{~F} \mathrm{Median} \\
\text { age: } 35\end{array}$ & $\begin{array}{l}\text { Abdominal pain; } \\
\text { hematuria; incidental } \\
\text { finding for hypertension } \\
\text { workup; other data } \\
\text { not available }\end{array}$ & $\begin{array}{l}\text { 1: SYT-SSX1; } \\
\text { 4: SYT-SSX2 }\end{array}$ & Radical nephrectomy & N/A \\
\hline 2 & $\begin{array}{l}\text { Kim DH et al.; } \\
2000[17]\end{array}$ & 2 & $\begin{array}{l}53 / M \\
47 / M\end{array}$ & $\begin{array}{l}\text { Rt flank pain } \\
\text { Rt flank pain, } \\
\text { gross hematuria }\end{array}$ & SYT-SSX2; & $\begin{array}{l}\text { Rt radical } \\
\text { nephrectomy; Rt } \\
\text { radical nephrectomy } \\
\text { with IVC } \\
\text { thrombectomy }\end{array}$ & $\begin{array}{l}\text { No recurrence } 6 \text { mons later; } \\
\text { lung mets } 5 \text { mons later, } \\
\text { death } 10 \text { mons post-op }\end{array}$ \\
\hline 3 & $\begin{array}{l}\text { Chen S et al.; } \\
2001 \text { [18] }\end{array}$ & 1 & $48 / \mathrm{M}$ & Hematuria & SYT-SSX 2 & $\begin{array}{l}\text { Lt radical } \\
\text { nephroureterectomy; } \\
\text { Radiation to surgical } \\
\text { bed; } 4 \text { cycles of } \\
\text { ifosfamide and } \\
\text { Doxorubicin }\end{array}$ & N/A \\
\hline 4 & $\begin{array}{l}\text { Koyama S } \\
\text { et al.; } 2001 \text { [8] }\end{array}$ & 1 & $47 / F$ & Right back pain & SYT-SSX 2 & $\begin{array}{l}\text { Rt radical } \\
\text { nephrectomy }\end{array}$ & $\begin{array}{l}\text { No recurrence } 17 \text { mons } \\
\text { later }\end{array}$ \\
\hline 5 & $\begin{array}{l}\text { Bella AJ et al.; } \\
2002 \text { [25] }\end{array}$ & 1 & $24 / M$ & Gross hematuria & $\begin{array}{l}\text { SYT-SSX } \\
\mathrm{t}(\mathrm{X} ; 18)\end{array}$ & $\begin{array}{l}\text { Rt radical } \\
\text { nephrectomy, } \\
\text { adjuvant } \\
\text { Actinomycin } \\
\text { + Vincristine }\end{array}$ & $\begin{array}{l}\text { No clinical evidence of } \\
\text { disease } 18 \text { mons after } \\
\text { nephrectomy }\end{array}$ \\
\hline 6 & $\begin{array}{l}\text { Dai YC et al.; } \\
2002[26]\end{array}$ & 1 & 19/F & $\begin{array}{l}\text { Abdominal Pain, } 3 \\
\text { mons of amenorrhea }\end{array}$ & $\begin{array}{l}\text { SYT-SSX } \\
\mathrm{t}(\mathrm{X} ; 18)\end{array}$ & Rt nephrectomy & $\begin{array}{l}\text { Recurrence of tumor in } \\
\text { retroperitoneum and } \\
\text { abdominal wall } 9 \text { mons } \\
\text { after surgery }\end{array}$ \\
\hline 7 & $\begin{array}{l}\text { Vesoulis Z } \\
\text { et al.; } 2003 \text { [27] }\end{array}$ & 1 & $38 / \mathrm{M}$ & $\begin{array}{l}\text { Acute abdominal } \\
\text { pain }\end{array}$ & SST-SSX1 & $\begin{array}{l}\text { Lt radical } \\
\text { nephrectomy }\end{array}$ & $\mathrm{N} / \mathrm{A}$ \\
\hline 8 & $\begin{array}{l}\text { Moch H et al.; } \\
2003 \text { [28] }\end{array}$ & 2 & $\begin{array}{l}47 / M \\
56 / F\end{array}$ & Renal mass & $\begin{array}{l}\text { SST-SSX1/ } \\
\text { SYT-SSX2 }\end{array}$ & $\begin{array}{l}\text { Nephrectomy/Rt } \\
\text { nephrectomy }\end{array}$ & $\begin{array}{l}\text { Local recurrence } 11 \text { years } \\
\text { later/ N/A }\end{array}$ \\
\hline 9 & $\begin{array}{l}\text { Chen PC et al.; } \\
2003 \text { [29] }\end{array}$ & 1 & 19/M & $\begin{array}{l}\text { left flank pain and } \\
\text { intermittent } \\
\text { hematuria }\end{array}$ & N/A & $\begin{array}{l}\text { Lt radical } \\
\text { nephrectomy + IVC } \\
\text { thrombectomy; } \\
\text { adjuvant Ifosfamide } \\
+ \text { Etoposide }\end{array}$ & $\begin{array}{l}\text { died of sepsis } 1 \text { month } \\
\text { after surgery }\end{array}$ \\
\hline 10 & $\begin{array}{l}\text { Park SJ et al.; } \\
2004 \text { [9] }\end{array}$ & 1 & $32 / F$ & $\begin{array}{l}\text { Intermittent } \\
\text { abdominal pain }\end{array}$ & N/A & $\begin{array}{l}\text { Lt radical } \\
\text { nephrectomy } \\
\text { + thormbectomy; } \\
6 \text { cycles Ifosfamide } \\
\text { + Doxorubicin }\end{array}$ & $\begin{array}{l}\text { Metastasis to lung } 4 \text { mons } \\
\text { post-op, complete remission } \\
\text { after chemotherapy }\end{array}$ \\
\hline 11 & $\begin{array}{l}\text { Jun SY } \mathrm{t} \text { al; } \\
2004[30]\end{array}$ & 3 & $\begin{array}{l}27,35 / F \\
26 / M\end{array}$ & Rt flank pain & SYT-SSX2 & $\begin{array}{l}\text { Rt radical } \\
\text { nephrectomies }\end{array}$ & $\begin{array}{l}\text { 1F: disease free } 5 \text { mons } \\
\text { post-op; } 2 \text { F: lumbar } \\
\text { vertebral mets } 5 \text { mons } \\
\text { post-op, } 6 \text { mons disease } \\
\text { free post-resection; } 3 \mathrm{M} \text { : } \\
\text { bilateral hemothorax; } \\
\text { death } 34 \text { days } \\
\text { post-op }\end{array}$ \\
\hline 12 & $\begin{array}{l}\text { Tornkvist M } \\
\text { et al.; } 2004 \text { [24] }\end{array}$ & 1 & $34 / F$ & N/A & SYT-SSX 2 & $\begin{array}{l}\text { Rt nephrectomy, } \\
\text { chemotherapy }\end{array}$ & $\begin{array}{l}\text { Visceral recurrence; } \\
\text { lung metastases }\end{array}$ \\
\hline 13 & $\begin{array}{l}\text { Schaal CH } \\
\text { et al.; } 2004 \text { [14] }\end{array}$ & 1 & $27 / M$ & $\begin{array}{l}\text { Hematuria and large } \\
\text { abdominal mass }\end{array}$ & $\mathrm{N} / \mathrm{A}$ & $\begin{array}{l}\text { Ifosfamide and } \\
\text { Adriamycin, followed } \\
\text { by Rt Radical } \\
\text { nephrectomy }\end{array}$ & $\begin{array}{l}\text { No recurrence after one } \\
\text { year }\end{array}$ \\
\hline 14 & $\begin{array}{l}\text { Shao L et al.; } \\
2004 \text { [31] }\end{array}$ & 4 & N/A & N/A & $\mathrm{N} / \mathrm{A}$ & $\mathrm{N} / \mathrm{A}$ & N/A \\
\hline
\end{tabular}


Table 1 List of 114 cases of renal SS published in the literature (Continued)

\begin{tabular}{|c|c|c|c|c|c|c|c|}
\hline $\begin{array}{l}\text { Case } \\
\text { Report/ } \\
\text { Series }\end{array}$ & $\begin{array}{l}\text { Author/Year } \\
\text { of publication }\end{array}$ & $\begin{array}{l}\text { No of } \\
\text { cases }\end{array}$ & $\begin{array}{l}\text { Age(Y)/Gender } \\
(\mathrm{M} / \mathrm{F})\end{array}$ & $\begin{array}{l}\text { Presenting } \\
\text { symptoms }\end{array}$ & $\begin{array}{l}\text { Fusion gene } \\
\text { Variant }\end{array}$ & Treatment & Outcome \\
\hline 15 & $\begin{array}{l}\text { Shannon BA } \\
\text { et al.; } 2005 \text { [32] }\end{array}$ & 1 & $60 / M$ & Hematuria & SYT-SSX 2 & $\begin{array}{l}\text { Rt radical } \\
\text { nephrectomy, } \\
\text { Imatinib, } 5 \text { cycles of } \\
\text { adjuvant chemotherapy }\end{array}$ & $\begin{array}{l}\text { Pulmonary metastasis } \\
6 \text { mons after surgery; } \\
\text { death } 12 \text { mons later }\end{array}$ \\
\hline 16 & $\begin{array}{l}\text { Perlmutter AE } \\
\text { et al.; } 2005 \text { [33] }\end{array}$ & 1 & $61 / F$ & $\begin{array}{l}\text { Right flank pain and } \\
\text { gross hematuria }\end{array}$ & SYT-SSX 2 & $\begin{array}{l}\text { Rt nephrectomy, } \\
\text { refused adjuvant } \\
\text { chemotherapy }\end{array}$ & $\begin{array}{l}\text { No recurrence } 5 \text { mons } \\
\text { post-surgery }\end{array}$ \\
\hline 17 & $\begin{array}{l}\text { Stage et al.; } \\
2005[34]\end{array}$ & 1 & $51 / F$ & $\begin{array}{l}\text { Renal masses } \\
\text { incidentally found }\end{array}$ & N/A & N/A & N/A \\
\hline 18 & $\begin{array}{l}\text { Paláu L MA } \\
\text { et al.; } 2007 \text { [35] }\end{array}$ & 1 & $71 / F$ & $\begin{array}{l}\text { Flank pain and } \\
\text { gross hematuria }\end{array}$ & SYT-SSX 2 & Lt nephrectomy & $\begin{array}{l}\text { Recovery in } 22 \text { mons } \\
\text { after surgery }\end{array}$ \\
\hline 19 & $\begin{array}{l}\text { Drozenova } \\
\text { et al.; } 2008 \text { [36] }\end{array}$ & 2 & $\begin{array}{l}33 / \mathrm{M} \\
57 / \mathrm{F}\end{array}$ & $\begin{array}{l}\text { Rt flank pain } \\
\text { Lt flank pain }\end{array}$ & $\begin{array}{l}\text { SYT-SSX1/ } \\
\text { SYT-SSX1 }\end{array}$ & $\begin{array}{l}\text { Rt Radical nephrectomy/ Lt } \\
\text { Radical nephrectomy }\end{array}$ & $\begin{array}{l}\text { Local recurrence and lung } \\
\text { mets; death } 6 \text { mons } \\
\text { later/ N/A }\end{array}$ \\
\hline 20 & $\begin{array}{l}\text { Mirza M et al.; } \\
2008 \text { [37] }\end{array}$ & 1 & $17 / \mathrm{M}$ & $\begin{array}{l}\text { Flank pain and } \\
\text { gross hematuria }\end{array}$ & SYT-SSX 2 & Lt radical nephrectomy & No recurrence 1 year later \\
\hline 21 & $\begin{array}{l}\text { Gabilondo F } \\
\text { et al.; } 2008 \text { [38] }\end{array}$ & 1 & $32 / F$ & $\begin{array}{l}\text { Mild abdominal pain; } \\
\text { gross hematuria }\end{array}$ & $\begin{array}{l}\text { SYT-SSX } \\
t(X ; 18)\end{array}$ & Rt radical nephrectomy & N/A \\
\hline 22 & $\begin{array}{l}\text { Zakhary MM } \\
\text { et al.; } 2008 \text { [39] }\end{array}$ & 1 & $52 / F$ & Right flank pain & N/A & Rt nephrectomy & N/A \\
\hline 23 & $\begin{array}{l}\text { Chung SD } \\
\text { et al.; } 2008 \text { [40] }\end{array}$ & 2 & $\begin{array}{l}30 / F \\
49 / F\end{array}$ & $\begin{array}{l}\text { Rt flank pain } \\
\text { Lt loin pain }\end{array}$ & SYT-SSX1 & $\begin{array}{l}\text { Rt radical } \\
\text { nephrectomy/ Lt } \\
\text { radical nephrectomy }\end{array}$ & $\begin{array}{l}\text { No recurrence } 15 \text { mons } \\
\text { post-op/ No recurrence } \\
27 \text { mons post-op }\end{array}$ \\
\hline 24 & $\begin{array}{l}\text { Erturhan S } \\
\text { et al.; } 2008 \text { [41] }\end{array}$ & 1 & $59 / \mathrm{M}$ & $\begin{array}{l}\text { Right lumbar pain } \\
\text { and palpable mass }\end{array}$ & N/A & N/A & $\mathrm{N} / \mathrm{A}$ \\
\hline 25 & $\begin{array}{l}\text { Divetia M et al.; } \\
2008 \text { [42] }\end{array}$ & 7 & $\begin{array}{l}2-M \\
5-F \\
\text { (15-46 years) }\end{array}$ & $\begin{array}{l}\text { Abdominal lump, } \\
\text { hematuria }\end{array}$ & $\begin{array}{l}3 \text { SYT-SSX1/ } \\
1 \text { SYT-SSX2 }\end{array}$ & Radical nephrectomy & $\begin{array}{l}\text { Lung mets in } 2 \text { patients; } \\
\text { death at } 6 \text { and } 12 \text { mons, } \\
\text { respectively }\end{array}$ \\
\hline 26 & $\begin{array}{l}\text { Dassi V et al.; } \\
2009 \text { [43] }\end{array}$ & 1 & $20 / F$ & Flank pain & $\begin{array}{l}\text { SYT-SSX } \\
t(X ; 18)\end{array}$ & Lt radical nephrectomy & N/A \\
\hline 28 & $\begin{array}{l}\text { Kawahara et al.; } \\
2009[44]\end{array}$ & 1 & $40 / F$ & Abdominal pain & SYT-SSX 1 & Radical nephrectomy & N/A \\
\hline 29 & $\begin{array}{l}\text { Long JA et al.; } \\
2009 \text { [45] }\end{array}$ & 3 & $\begin{array}{l}\text { (Age range: } \\
27-33 \text { years) }\end{array}$ & $\begin{array}{l}\text { Back pain and } \\
\text { spontaneous } \\
\text { rupture }\end{array}$ & $\begin{array}{l}\text { SYT-SSX } \\
\mathrm{t}(\mathrm{X} ; 18)\end{array}$ & $\begin{array}{l}2 \text { Rt radical } \\
\text { nephrectomy; } 1 \mathrm{Lt} \\
\text { radical nephrectomy }\end{array}$ & $\begin{array}{l}2 \text { patients: total remission } \\
25 \text { mons post-op; } 1 \text { patient: } \\
\text { death } 24 \text { mons post-op }\end{array}$ \\
\hline 30 & $\begin{array}{l}\text { Wezel F et al.; } \\
2010 \text { [46] }\end{array}$ & 1 & $47 / M$ & $\begin{array}{l}\text { Hematuria, abdominal } \\
\text { pain, weight loss }\end{array}$ & $\begin{array}{l}\text { SYT-SSX } \\
t(X ; 18)\end{array}$ & Nephrectomy & $\begin{array}{l}\text { No recurrence } 18 \text { weeks } \\
\text { after surgery }\end{array}$ \\
\hline 31 & $\begin{array}{l}\text { Wang Z-H } \\
\text { et al.; } 2009 \text { [47] }\end{array}$ & 4 & $\begin{array}{l}2 / \mathrm{F} \\
2 / \mathrm{M} \\
32 \text { to } 48 \text { years }\end{array}$ & $\begin{array}{l}\text { Low back pain, } \\
\text { hematuria }\end{array}$ & SYT-SSX1 & $\begin{array}{l}\text { Radical nephrectomy; } \\
3 \text { Lt side, } 1 \text { Rt side }\end{array}$ & $\begin{array}{l}\text { Liver + lung metastasis; } \\
\text { death at 5, 8, 18, and } \\
21 \text { mons post-op, } \\
\text { respectively }\end{array}$ \\
\hline 32 & $\begin{array}{l}\text { Kageyama S } \\
\text { et al.; } 2010 \text { [48] }\end{array}$ & 1 & $67 / M$ & $\begin{array}{l}\text { Gross hematuria } \\
\text { and right flank pain }\end{array}$ & SYT-SSX 2 & $\begin{array}{l}\text { Rt nephroureterectomy; } \\
\text { Ifosfamide and } \\
\text { Etoposide regimen }\end{array}$ & $\begin{array}{l}\text { Tumor recurrence } 33 \text { mons } \\
\text { post nephrectomy; } \\
\text { Liver mets; death } \\
4 \text { years later }\end{array}$ \\
\hline 33 & $\begin{array}{l}\text { Tan YS et al.; } \\
2010[49]\end{array}$ & 4 & N/A & N/A & N/A & N/A & N/A \\
\hline 34 & $\begin{array}{l}\text { Romero-Rojas } \\
\text { AE et al.; } \\
2013 \text { [50] }\end{array}$ & 1 & $15 / \mathrm{M}$ & $\begin{array}{l}\text { Lt abdominal pain; } \\
\text { weight loss }\end{array}$ & N/A & $\begin{array}{l}\text { Neoadjuvant } \\
\text { chemotherapy } \\
\text { followed by Lt radical } \\
\text { nephrectomy }\end{array}$ & Death 1.8 years later \\
\hline 35 & $\begin{array}{l}\text { Lakshmaiah KC } \\
\text { et al.; } 2010[51]\end{array}$ & 2 & $\begin{array}{l}50 / F \\
45 / M\end{array}$ & $\begin{array}{l}\text { Rt flank pain/ } \\
\text { Lt flank pain, }\end{array}$ & $\begin{array}{l}\text { SYT-SSX2/ } \\
\text { NOT DONE }\end{array}$ & Radical nephrectomy & $\begin{array}{l}\text { No recurrence } 2 \text { years } \\
\text { post-op/ lost to follow-up }\end{array}$ \\
\hline
\end{tabular}


Table 1 List of 114 cases of renal SS published in the literature (Continued)

\begin{tabular}{|c|c|c|c|c|c|c|c|}
\hline $\begin{array}{l}\text { Case } \\
\text { Report/ } \\
\text { Series }\end{array}$ & $\begin{array}{l}\text { Author/ Year } \\
\text { of publication }\end{array}$ & $\begin{array}{l}\text { No of } \\
\text { cases }\end{array}$ & $\begin{array}{l}\text { Age(Y)/Gender } \\
(\mathrm{M} / \mathrm{F})\end{array}$ & $\begin{array}{l}\text { Presenting } \\
\text { symptoms }\end{array}$ & $\begin{array}{l}\text { Fusion gene } \\
\text { Variant }\end{array}$ & Treatment & Outcome \\
\hline 36 & $\begin{array}{l}\text { Kataria et al.; } \\
2010[52]\end{array}$ & 1 & $52 / F$ & Renal mass & SYT-SSX 2 & $\begin{array}{l}\text { Radical nephrectomy } \\
\text { IVC thrombectomy; } \\
\text { adjuvant chemo-radiation }\end{array}$ & Mets to lung \\
\hline 37 & $\begin{array}{l}\text { Grampurohit } \\
\text { VU et al.; } \\
2011 \text { [53] }\end{array}$ & 1 & $21 / F$ & $\begin{array}{l}\text { Fever, hematuria; } \\
\text { right flank pain }\end{array}$ & $\begin{array}{l}\text { SYT-SSX } \\
\mathrm{t}(\mathrm{X} ; 18)\end{array}$ & Rt nephrectomy & $\begin{array}{l}\text { No recurrence } 6 \text { mons } \\
\text { post-surgery }\end{array}$ \\
\hline 38 & $\begin{array}{l}\text { Ozkan EE et al.; } \\
2011 \text { [20] }\end{array}$ & 1 & $68 / F$ & $\begin{array}{l}\text { Right flank pain and } \\
\text { abdominal distention }\end{array}$ & N/A & $\begin{array}{l}\text { Rt nephroureterectomy, } \\
4 \text { cycles Ifosfamide } \\
\text { and Doxorubicin }\end{array}$ & $\begin{array}{l}\text { No recurrence one year } \\
\text { later }\end{array}$ \\
\hline 39 & $\begin{array}{l}\text { Karafin M et al.; } \\
2011 \text { [54] }\end{array}$ & 3 & $\begin{array}{l}39 / \mathrm{F} \\
41 / \mathrm{M} \\
53 / \mathrm{M}\end{array}$ & N/A & $\begin{array}{l}\text { SYT-SSX2 } \\
\text { SYT-SSX2 } \\
\text { N/A }\end{array}$ & N/A & N/A \\
\hline 40 & $\begin{array}{l}\text { Nishida T et al.; } \\
2011 \text { [55] }\end{array}$ & 1 & $63 / F$ & Dysuria, hematuria & $\begin{array}{l}\text { SYT-SSX } 1 \& \\
2\end{array}$ & Rt Radical nephrectomy & $\begin{array}{l}\text { No recurrence one year } \\
\text { postop }\end{array}$ \\
\hline 41 & $\begin{array}{l}\text { Pitino A et al.; } \\
2011 \text { [8] }\end{array}$ & 1 & $67 / M$ & $\begin{array}{l}\text { Lumbar pain, } \\
\text { gross hematuria }\end{array}$ & SYT-SSX 2 & $\begin{array}{l}\text { Lt Nephroureterectomy; } \\
\text { adjuvant Epirubicin } \\
\text { post-op }\end{array}$ & $\begin{array}{l}\text { Local recurrence of disease } \\
24 \text { mons post- surgery }\end{array}$ \\
\hline 42 & $\begin{array}{l}\text { Bakhshi et al.; } \\
2012 \text { [56] }\end{array}$ & 1 & $33 / F$ & $\begin{array}{l}\text { Abdominal pain and } \\
\text { gross hematuria }\end{array}$ & SYT-SSX 2 & $\begin{array}{l}\text { Lt radical nephrectomy; } \\
\text { external radiotherapy }\end{array}$ & No recurrence at 2 years \\
\hline 43 & $\begin{array}{l}\text { Lopes et al.; } \\
2013 \text { [3] }\end{array}$ & 1 & $19 / \mathrm{M}$ & $\begin{array}{l}\text { Lumbar pain, } \\
\text { gross hematuria }\end{array}$ & $\begin{array}{l}\text { Negative } \\
\text { translocation }\end{array}$ & $\begin{array}{l}\text { Lt nephrectomy, } \\
\text { thrombectomy; } \\
5 \text { cycles of } \\
\text { doxorubicin }\end{array}$ & $\begin{array}{l}\text { Lung mets several mons } \\
\text { post-op }\end{array}$ \\
\hline 44 & $\begin{array}{l}\text { Pereira E Silva } \\
\text { R et al.; } 2013 \\
\text { [57] }\end{array}$ & 1 & $17 / M$ & $\begin{array}{l}\text { Incidental large renal } \\
\text { mass after workup for } \\
\text { secondary } \\
\text { hypertension }\end{array}$ & $\begin{array}{l}\text { Negative } \\
\text { translocation }\end{array}$ & $\begin{array}{l}\text { Radical nephrectomy } \\
\text { followed by ifosfamide }\end{array}$ & $\begin{array}{l}\text { No recurrence } 29 \text { mons } \\
\text { later }\end{array}$ \\
\hline 45 & $\begin{array}{l}\text { Marković-Lipkovski J } \\
\text { et al.; } 2013 \text { [12] }\end{array}$ & 1 & $38 / \mathrm{M}$ & $\begin{array}{l}\text { Rt flank pain; } \\
\text { fever; hematuria }\end{array}$ & SYT-SSX2 & Rt radical nephrectomy & Died three mons later \\
\hline 46 & $\begin{array}{l}\text { Moorthy et al.; } \\
2014 \text { [58] }\end{array}$ & 1 & $46 / \mathrm{M}$ & Flank pain & SYT-SSX 2 & Lt radical nephrectomy & N/A \\
\hline 47 & $\begin{array}{l}\text { Majumber } \\
\text { et al.; } 2014 \text { [59] }\end{array}$ & 1 & $46 / F$ & $\begin{array}{l}\text { Flank pain, } \\
\text { hematuria }\end{array}$ & $\mathrm{N} / \mathrm{A}$ & Rt radical nephrectomy & $\begin{array}{l}\text { No evidence of disease } \\
\text { after } 2 \text { mons follow up }\end{array}$ \\
\hline 48 & $\begin{array}{l}\text { Schoolmeester } \\
\text { JK et al.; } \\
2014 \text { [60] }\end{array}$ & 16 & $\begin{array}{l}\text { 9M/7F } \\
\text { 17-78 yrs.; } \\
\text { Median: } 46 \text { yrs }\end{array}$ & N/A; Rt: 10; Lt: 6; & $\begin{array}{l}\text { SYT-SSX2: } \\
\text { 10; SYT- } \\
\text { SSX1: 5; } \\
\text { 1: failed }\end{array}$ & $\begin{array}{l}\text { 14: Radical nephrectomy; } \\
\text { 1: partial nephrectomy; } \\
\text { 1: needle biopsy }\end{array}$ & $\begin{array}{l}\text { 6: death within 1-58 mons } \\
\text { (mean } 31 \text { mons); } 5 \text { : no } \\
\text { recurrence } 12-77 \text { mons } \\
\text { ( } 39 \text { mons); } 1 \text { : alive with } \\
\text { spine mets } 11 \text { mons later }\end{array}$ \\
\hline 49 & $\begin{array}{l}\text { Kim MS et al.; } \\
2014 \text { [61] }\end{array}$ & 1 & $38 / F$ & Lt flank pain & SYT-SSX2 & $\begin{array}{l}\text { Lap Lt radical } \\
\text { nephrectomy followed } \\
\text { by radiation to } \\
\text { surgical bed }\end{array}$ & $\begin{array}{l}\text { Recurrence at the distal } \\
\text { ureter and uretero-vesical } \\
\text { junction } 6 \text { mons } \\
\text { post- surgery }\end{array}$ \\
\hline 50 & $\begin{array}{l}\text { Ozkanli SS } \\
\text { et al.; } 2014 \text { [62] }\end{array}$ & 1 & $45 / \mathrm{M}$ & $\begin{array}{l}\text { Flank pain; } \\
\text { macroscopic } \\
\text { hematuria }\end{array}$ & $\begin{array}{l}\text { SYT-SSX } \\
\mathrm{t}(\mathrm{X} ; 18)\end{array}$ & Lt radical nephrectomy & $\mathrm{N} / \mathrm{A}$ \\
\hline 51 & $\begin{array}{l}\text { Mishra S et al.; } \\
2015 \text { [13] }\end{array}$ & 1 & $60 / M$ & $\begin{array}{l}\text { Flank pain, } \\
\text { hematuria }\end{array}$ & $\begin{array}{l}\text { SYT-SSX } \\
t(X ; 18)\end{array}$ & Radical nephrectomy & N/A \\
\hline 52 & $\begin{array}{l}\text { Wang Z et al.; } \\
2015 \text { [61] }\end{array}$ & 1 & $54 / F$ & $\begin{array}{l}\text { Flank pain, } \\
\text { hematuria }\end{array}$ & SYT-SSX 1 & Radical nephrectomy & $\begin{array}{l}\text { No recurrence } 12 \text { mons } \\
\text { post-surgery }\end{array}$ \\
\hline 53 & $\begin{array}{l}\text { Vedana M } \\
\text { et al.; } 2015 \text { [63] }\end{array}$ & 1 & $76 / F$ & $\begin{array}{l}\text { Flank pain; } \\
\text { hematuria }\end{array}$ & $\begin{array}{l}\text { SYT-SSX } \\
\mathrm{t}(\mathrm{X} ; 18)\end{array}$ & $\begin{array}{l}\text { Rt radical } \\
\text { nephro-ureterectomy }\end{array}$ & $\begin{array}{l}\text { No recurrence } 20 \text { mons } \\
\text { post-surgery }\end{array}$ \\
\hline
\end{tabular}


Table 1 List of 114 cases of renal SS published in the literature (Continued)

\begin{tabular}{|c|c|c|c|c|c|c|c|}
\hline $\begin{array}{l}\text { Case } \\
\text { Report/ } \\
\text { Series }\end{array}$ & $\begin{array}{l}\text { Author/ Year } \\
\text { of publication }\end{array}$ & $\begin{array}{l}\text { No of } \\
\text { cases }\end{array}$ & $\begin{array}{l}\text { Age }(Y) / \text { Gender } \\
(\mathrm{M} / \mathrm{F})\end{array}$ & $\begin{array}{l}\text { Presenting } \\
\text { symptoms }\end{array}$ & $\begin{array}{l}\text { Fusion gene } \\
\text { Variant }\end{array}$ & Treatment & Outcome \\
\hline 54 & $\begin{array}{l}\text { Lv X-F et al.; } \\
2015 \text { [64] }\end{array}$ & 5 & $\begin{array}{l}\text { 2F/3M } \\
\text { (15-43 yrs.; } \\
\text { Median: } \\
27.4 \text { yrs) }\end{array}$ & N/A & N/A & N/A & N/A \\
\hline 55 & $\begin{array}{l}\text { Present case El } \\
\text { Chediak A. et al.; } \\
2016\end{array}$ & 1 & $26 / M$ & Rt flank pain; hematuria & SYT-SSX2 & $\begin{array}{l}\text { Rt radical nephrectomy, } \\
\text { Doxorubicin Ifosfamide }\end{array}$ & $\begin{array}{l}\text { Lung metastasis } 6 \text { mons } \\
\text { post nephrectomy; no } \\
\text { recurrence one year post } \\
\text { chemotherapy }\end{array}$ \\
\hline
\end{tabular}

M Males, F Females, Yrs Years, Rt Right, Lt Left, Mets Metastasis, Mons Months, IVC Inferior vena cava, N/A Not Applicable

transcript had a significantly longer metastasis-free survival [15]. On the contrary, Japanese patients, with synovial sarcoma, having positive SYT-SSX fusion transcript, were retrospectively analyzed [17]. They concluded that SYT-SSX fusion type was not found to be a significant prognostic factor, unlike tumor size and histological grading, for patients with localized synovial sarcoma [17]. Another study by Guillou et al. also confirmed that histologic grading, and not SYT-SSX fusion type, was a stronger predictor of survival, by collecting retrospective data on 165 SS patients [18].

In our sample, staging information was available for 46 patients, based on the 7th edition TNM staging for soft tissue sarcomas. Among the patients having the SYT-SSX2 fusion protein. $53.8 \%$ were stage II and $34.6 \%$ were of stage III. 33 and $25 \%$ of patients with SYT-SSX1 transcript were stage II and III, respectively. $25 \%$ of patients with SYT-SSX1 were of stage IV, versus only $7.7 \%$ for SYT-SSX2 patients.

Lungs were the most common metastatic site, regardless of the fusion type. However, $50 \%$ of patients with SYTSSX2 fusion type had metastasis to the liver. Although lungs and liver are common sites for metastasis for renal SS $[19,20]$, it was not reported before whether there is a relation between the site of metastasis and the type of fusion transcript. From the above, it appears that SYT-SSX 1 behaves more aggressively. However, studies with a larger number of patients and longer follow-up periods are needed to verify these observations, especially in the light of the contradicting data, presenting on the prognostic value of the SYT-SSX fusion protein.

Although SS is considered an aggressive form of STS where metastasis can occur in $50 \%$ of the cases, it was found to be sensitive to Anthracycline based chemotherapy [21]. However, due to the rarity of the tumor, a standard therapy has not been established. Treatment modalities include surgical resection and chemotherapy. A combination of chemotherapy (Ifosfamide and Doxorubicin) and surgery has yielded positive results $[6,9,12,13]$. Based on our review of the literature, 10 patients took Ifosfamide and Doxorubicin, either together or in combination with other chemotherapeutic agents. 5 out of 10 cases were reported to have complete remission. This further corroborates the effectiveness of giving Ifosfamide and Doxorubicin as a regimen to treat primary renal SS. The basis for chemotherapy was tumor volume reduction, mainly attributed to Ifosfamide. In one case report, the combination of Ifosfamide and Doxorubicin lead to a 50\% reduction of the tumor before consequent resection [14]. The controversy of the impact of adjuvant chemotherapy on overall survival, in SS patients, is limited by randomized clinical trials'(RCTs) sample size and varied chemotherapy regimens with discrepant results [22]. The Sarcoma Meta-analysis Collaboration (SMAC) group performed a meta-analysis of all known randomized clinical trials in 1997. Their results indicated that doxorubicinbased chemotherapy served to significantly improve time to local and distant recurrence, as well as overall recurrence-free survival in comparison to patients who were just observed [23]. An increase in overall survival was not statistically significant [23]. Another metaAnalysis of RCTs of adjuvant chemotherapy for localized resectable STS was conducted by Pervais et al. where they built on the results of the SMAC study and narrowed the confidence intervals [24]. This meta-analysis demonstrated marginal efficacy of doxorubicin based chemotherapy with respect to local recurrence, distant recurrence, overall recurrence, and overall survival, in comparison to those who did not receive adjuvant chemotherapy [22].

\section{Conclusion}

In conclusion, primary SS of the kidney is an aggressive rare disease that can be mistaken for other types of renal cell carcinomas. Its diagnosis is based on morphological and molecular studies demonstrating spindle cells and the SYT-SSX translocation. However, establishing a correct diagnosis may be difficult. Prognosis can be enhanced by use of anthracycline based chemotherapy. Moreover, the combination of surgery and chemotherapy has shown positive results. Particularly, we propose the use of Ifosfamide and Doxorubicin as a standard chemotherapy to induce complete remission. Since the disease may have rapid course with unfavorable outcomes, clinicians need to be aware of the existence of this rare entity, so that timely and appropriate therapy can be initiated. 


\section{Abbreviations}

$\mathrm{CT}$ : Computer tomography; FISH: fluorescence in situ hybridization; IVC: Inferior vena cava; MRI: Magnetic resonance imaging; N/A: Not available; RCT: Randomized clinical trials; RT-PCR: reverse transcriptase-polymerase chain reaction; SS: Synovial sarcoma; STS: Soft tissue sarcoma

\section{Funding}

No funding from an external party was received. Genetic testing was conducted using the corresponding author's research fund.

\section{Availability of data and materials}

All data generated and analyzed during this study are included in this published article.

\section{Authors' contributions}

EA was involved in acquisition, analysis, and interpretation of data (literature), and manuscript writing. SN and SS performed histological examination of the resected tumor and provided the pathological images in our manuscript. MR performed molecular analysis of the tumor sample. Both MD and TS were involved in manuscript writing and editing. SA was involved in data analysis, interpretation of data (literature), and revising the manuscript. He also gave final approval of the version prior to submission. All authors read and approved the final manuscript.

\section{Ethics approval and consent to participate}

As this is a case report, the Institutional Review Board at the American University of Beirut waivers any approval.

\section{Consent for publication}

A written informed consent has been obtained from the patient, and is available to the Editor-in-chief upon request.

\section{Competing interests}

The authors declare that they have no competing interests.

\section{Publisher's Note}

Springer Nature remains neutral with regard to jurisdictional claims in published maps and institutional affiliations.

\section{Author details}

'Department of Internal Medicine, Division of Hematology/Oncology, American University of Beirut - Medical Center, P.o.Box: 11-0236, Riad El Solh, Beirut 110 72020, Lebanon. ${ }^{2}$ Department of Pathology and Laboratory Medicine, American University of Beirut - Medical Center, Beirut, Lebanon.

Received: 14 November 2016 Accepted: 3 May 2018

Published online: 11 May 2018

\section{References}

1. Fletcher CD. The evolving classification of soft tissue tumours - an update based on the new 2013 WHO classification. Histopathology. 2014;64(1):2-11.

2. Goldblum JR, Weiss SW, Andrew L. Folpe Enzinger and Weiss's soft tissue tumors. sixth ed. St Louis, Missouri: Saunders; 2001. 1176.

3. Lopes $\mathrm{H}$, et al. Primary monophasic synovial sarcoma of the kidney: a case report and review of literature. Clin Med Insights Oncol. 2013;7:257-62.

4. Spillane AJ, et al. Synovial sarcoma: a clinicopathologic, staging, and prognostic assessment. J Clin Oncol. 2000;18(22):3794-803.

5. Argani P. et al. Primary renal synovial sarcoma: molecular and morphologic delineation of an entity previously included among embryonal sarcomas of the kidney. Am J Surg Pathol. 2000;24(8):1087-96.

6. Kohle O, et al. Soft tissue sarcomas of the kidney. Rare Tumors. 2015;7(1):5635.

7. Namita Chittoria, B.IR. Renal Cell Carcinoma. 2013 [cited 2016; Available from: http://www.clevelandclinicmeded.com/medicalpubs/diseasemanagement/ nephrology/renal-cell-carcinoma/\#top. Accessed 25 May 2017.

8. Pitino A, et al. Primary synovial sarcoma of the kidney. A case report with pathologic appraisal investigation and literature review. Pathologica. 2011 103(5):271-8.

9. Park SJ, et al. A case of renal synovial sarcoma: complete remission was induced by chemotherapy with doxorubicin and ifosfamide. Korean J Intern Med. 2004;19(1):62-5.
10. Deshmukh R, Mankin HJ, Singer S. Synovial sarcoma: the importance of size and location for survival. Clin Orthop Relat Res. 2004;419:155-61.

11. Singer $\mathrm{S}$, et al. Synovial sarcoma: prognostic significance of tumor size, margin of resection, and mitotic activity for survival. J Clin Oncol. 1996;14(4): 1201-8.

12. Markovic-Lipkovski J, et al. Rapidly progressive course of primary renal synovial sarcoma-case report. Srp Arh Celok Lek. 2013;141(11-12):814-8.

13. Mishra S, et al. Primary synovial sarcoma of the kidney. Saudi J Kidney Dis Transpl. 2015;26(5):996-9.

14. Schaal $\mathrm{CH}$, Navarro FC, Moraes Neto FA. Primary renal sarcoma with morphologic and immunohistochemical aspects compatible with synovial sarcoma. Int Braz J Urol. 2004;30(3):210-3.

15. Ladanyi M. Fusions of the SYT and SSX genes in synovial sarcoma. Oncogene. 2001;20(40):5755-62.

16. Kawai A, et al. SYT-SSX gene fusion as a determinant of morphology and prognosis in synovial sarcoma. N Engl J Med. 1998;338(3):153-60.

17. Kim DH, et al. Primary synovial sarcoma of the kidney. Am J Surg Pathol. 2000;24(8):1097-104

18. Chen S, et al. Primary synovial sarcoma of the kidney: a case report with literature review. Int J Surg Pathol. 2001;9(4):335-9.

19. lacovelli $R$, et al. Clinical and pathological features of primary renal synovial sarcoma: analysis of 64 cases from 11 years of medical literature. BJU Int. 2012:110(10):1449-54.

20. Ozkan EE, Mertsoylu H, Ozardali HI. A case of renal synovial sarcoma treated with adjuvant ifosfamide and doxorubicin. Intern Med. 2011;50(15):1575-80.

21. Karavasilis V, Seddon BM, Ashley S, Al-Muderis O, Fisher C, Judson I. Significant clinical benefit of first-line palliative chemotherapy in advanced soft-tissue sarcoma: retrospective analysis and identification of prognostic factors in 488 patients. Cancer. 2008;112(7):1585-91. https://www.ncbi.nlm nih.gov/pubmed/18278813.

22. Pervaiz $\mathrm{N}$, et al. A systematic meta-analysis of randomized controlled trials of adjuvant chemotherapy for localized resectable soft-tissue sarcoma. Cancer. 2008;113(3):573-81.

23. Adjuvant chemotherapy for localised resectable soft tissue sarcoma in adults. Cochrane Database Syst Rev, 2000(4): p. Cd001419.

24. Tornkvist M, et al. A novel case of synovial sarcoma of the kidney: impact of SS18/SSX analysis of renal hemangiopericytoma-like tumors. Diagn Mol Pathol. 2004:13(1):47-51.

25. Bella AJ, Winquist EW, Perlman EJ. Primary synovial sarcoma of the kidney diagnosed by molecular detection of SYT-SSX fusion transcripts. J Urol. 2002;168(3):1092-3.

26. Dai $Y C$, et al. A rare synovial sarcoma of the kidney exhibiting translocation $(X ; 18)$ and SYT-SSX2 fusion gene. Zhonghua Yi Xue Za Zhi (Taipei). 2002; 65(6):293-7.

27. Vesoulis Z, et al. Fine needle aspiration biopsy of primary renal synovial sarcoma. A case report. Acta Cytol. 2003;47(4):668-72.

28. Moch $\mathrm{H}$, et al. Primary renal synovial sarcoma. A new entity in the morphological spectrum of spindle cell renal tumors. Pathologe. 2003;24(6): $466-72$.

29. Chen PC, et al. Primary renal synovial sarcoma with inferior vena cava and right atrium invasion. Int J Urol. 2003;10(12):657-60.

30. Jun SY, et al. Synovial sarcoma of the kidney with rhabdoid features: report of three cases. Am J Surg Pathol. 2004:28(5):634-7.

31. Shao L, Hill DA, Perlman EJ. Expression of WT-1, BCl-2, and CD34 by primary renal spindle cell tumors in children. Pediatr Dev Pathol. 2004:7(6):577-82.

32. Shannon BA, Murch A, Cohen RJ. Primary renal synovial sarcoma confirmed by cytogenetic analysis: a lesion distinct from sarcomatoid renal cell carcinoma. Arch Pathol Lab Med. 2005;129(2):238-40

33. Perlmutter $A E$, et al. Primary synovial sarcoma of the kidney. Int J Urol. 2005. 12(8):760-2

34. Stage AC, Pollock RE, Matin SF. Bilateral metastatic renal synovial sarcoma. Urology. 2005;65(2):389.

35. Palau LM, et al. Primary synovial sarcoma of the kidney with rhabdoid features. Int J Surg Pathol. 2007:15(4):421-8.

36. Drozenova J, et al. Primary synovial sarcoma of the kidney. Cesk Patol. 2008; 44(1):20-2.

37. Mirza M, Zamilpa I, Bunning J. Primary renal synovial sarcoma. Urology. 2008:72(3):716 e11-2

38. Gabilondo F, et al. Primary synovial sarcoma of the kidney: corroboration with in situ polymerase chain reaction. Ann Diagn Pathol. 2008:12(2):134-7. 
39. Zakhary MM, et al. Magnetic resonance imaging features of renal synovial sarcoma: a case report. Cancer Imaging. 2008;8:45-7.

40. Chung, S.D., et al., Primary synovial sarcoma of the kidney. J Formos Med Assoc, 2008. 107(4): 344-347.

41. Erturhan S, et al. Primary synovial sarcoma of the kidney: use of PET/CT in diagnosis and follow-up. Ann Nucl Med. 2008;22(3):225-9.

42. Divetia M, et al. Synovial sarcoma of the kidney. Ann Diagn Pathol. 2008; 12(5):333-9.

43. Dassi V, et al. Primary synovial sarcoma of kidney: a rare tumor with an atypical presentation. Indian J Urol. 2009;25(2):269-71.

44. Kawahara T, et al. Primary synovial sarcoma of the kidney. Case Rep Oncol. 2009;2(3):189-93.

45. Long JA, et al. Primitive renal synovial sarcoma: a cystic tumor in young patients. Prog Urol. 2009;19(7):474-8.

46. Wezel F, et al. Primary biphasic synovial sarcoma of the kidney. Urologe A. 2010;49(3):411-4.

47. Wang ZH, Wang XC, Xue M. Clinicopathologic analysis of 4 cases of primary renal synovial sarcoma. Chin J Cancer. 2010;29(2):212-6.

48. Kageyama $\mathrm{S}$, et al. Primary synovial sarcoma arising from a crossed ectopic kidney with fusion. Int J Urol. 2010;17(1):96-8.

49. Tan YS, et al. Synovial sarcoma of the kidney: a report of 4 cases with pathologic appraisal and differential diagnostic review. Anal Quant Cytol Histol. 2010;32(4):239-45.

50. Romero-Rojas AE, et al. Early age renal synovial sarcoma. Arch Esp Urol. 2010;63(6):464-71.

51. Lakshmaiah KC, et al. Primary synovial sarcoma of kidney-a report of 2 cases and review of literature. J Egypt Natl Canc Inst. 2010;22(3):149-53.

52. Kataria T, et al. Pulmonary metastasis from renal synovial sarcoma treated by stereotactic body radiotherapy: a case report and review of the literature. J Cancer Res Ther. 2010;6(1):75-9.

53. Grampurohit VU, Myageri A, Rao RV. Primary renal synovial sarcoma. Uro Ann. 2011;3(2):110-3.

54. Karafin $\mathrm{M}$, et al. Diffuse expression of PAX2 and PAX8 in the cystic epithelium of mixed epithelial stromal tumor, angiomyolipoma with epithelial cysts, and primary renal synovial sarcoma: evidence supporting renal tubular differentiation. Am J Surg Pathol. 2011;35(9):1264-73.

55. Nishida T, et al. Monophasic primary renal synovial sarcoma accompanied with a hemorrhagic cyst. Urol J. 2011;8(3):244-7.

56. Bakhshi GD, et al. Primary renal synovial sarcoma. Clin Pract. 2012;2(2):e44

57. Pereira ESR, et al. Primary synovial sarcoma of the kidney with unusual follow up findings. Can J Urol. 2013;20(2):6734-6.

58. Moorthy HK, Pillai BS, Varghese J. Primary renal synovial sarcoma: an oncologic surprise. Urol Case Rep. 2014;2(5):152-3.

59. Majumder A, et al. Primary renal synovial sarcoma: a rare tumor with an atypical presentation. Arch Iran Med. 2014;17(10):726-8

60. Schoolmeester JK, Cheville JC, Folpe AL. Synovial sarcoma of the kidney: a clinicopathologic, immunohistochemical, and molecular genetic study of 16 cases. Am J Surg Pathol. 2014:38(1):60-5.

61. Wang $Z$, et al. Primary synovial sarcoma of the kidney: a case report. Onco Lett. 2015;10(6):3542-4.

62. Ozkanli SS, et al. Primary synovial sarcoma of the kidney. Urol Int. 2014;92(3): 369-72.

63. Vedana $\mathrm{M}$, et al. Primary synovial cell sarcoma of the kidney: case report and review of the literature. Case Reports in Oncology. 2015;8(1):128-32.

64. LV XF, et al. Primary renal synovial sarcoma: computed tomography imaging findings. Acta Radiol. 2015;56(4):493-9.

\section{Ready to submit your research? Choose BMC and benefit from:}

- fast, convenient online submission

- thorough peer review by experienced researchers in your field

- rapid publication on acceptance

- support for research data, including large and complex data types

- gold Open Access which fosters wider collaboration and increased citations

- maximum visibility for your research: over $100 \mathrm{M}$ website views per year

At BMC, research is always in progress.

Learn more biomedcentral.com/submissions 